Editorial

\title{
Announcement of "International Congress on Food Science and Public Health" in Tehran, Iran
}

Program: International Congress on Food Science and Public Health

Location: Tehran, Iran

Congress main topics: New technologies at food processing and food safety; Nanotechnology in the food industry; Strategies to reduce energy consumption in the food industry; Food quality control and quality assurance; Prebiotic and probiotic foods; Risk assessment in the food industry; Food hygiene and nutrition during COVID-19; Food packaging and preservation; Food by-product utilization; Detection of food fraud; Food safety at home; Food toxicology

Congress date: June 2021

Website: www.tekmaconf.com

Email: info@tekmaconf.com

Tel.: +982166934275 